\title{
Correction to: Homeless Street Outreach: Spark for the Journey to a Dignified Life
}

\author{
Linda Plitt Donaldson and Wonhyung Lee
}

\section{Correction to:}

Chapter 14 in: H. Larkin et al. (eds.), Homelessness Prevention and Intervention in Social Work, https://doi.org/10.1007/978-3-030-03727-7_14

The published version of this book missed to include the following citation: Ellen Bassuk (1994). This has been updated. 